\title{
Structure of the exact wave function. II. Iterative configuration interaction method
}

$\operatorname{AUTHOR}(\mathrm{S}):$

Nakatsuji, H; Davidson, ER

\section{CITATION:}

Nakatsuji, H ...[et al]. Structure of the exact wave function. II. Iterative configuration interaction method. JOURNAL OF CHEMICAL PHYSICS 2001, 115(5): 2000-2006

\section{ISSUE DATE:}

2001-08-01

URL:

http://hdl.handle.net/2433/39732

\section{RIGHT:}

Copyright 2001 American Institute of Physics. This article may be downloaded for personal use only. Any other use requires prior permission of the author and the American Institute of Physics. 


\title{
Structure of the exact wave function. II. Iterative configuration interaction method
}

\author{
Hiroshi Nakatsuji ${ }^{\text {a) }}$ \\ Department of Synthetic Chemistry and Biological Chemistry, Graduate School of Engineering, \\ Kyoto University, Sakyo-ku, Kyoto 606-8501, Japan \\ Ernest R. Davidson \\ Department of Chemistry, Indiana University, Bloomington, Indiana 47405-6040
}

(Received 28 November 2000; accepted 10 May 2001)

\begin{abstract}
This is the second progress report on the study of the structure of the exact wave function. First, Theorem II of Paper I (H. Nakatsuji, J. Chem. Phys. 113, 2949 (2000)) is generalized: when we divide the Hamiltonian of our system into $N_{\mathrm{D}}$ (number of division) parts, we correspondingly have a set of $N_{\mathrm{D}}$ equations that is equivalent to the Schrödinger equation in the necessary and sufficient sense. Based on this theorem, the iterative configuration interaction (ICI) method is generalized so that it gives the exact wave function with the $N_{\mathrm{D}}$ number of variables in each iteration step. We call this the ICIND method. The ICIGSD (general singles and doubles) method is an important special case in which the GSD number of variables is involved. The ICI methods involving only one variable [ICION(one) or S(simplest)ICI] and only general singles (GS) number of variables (ICIGS) are also interesting. ICIGS may be related to the basis of the density functional theory. The convergence rate of the ICI calculations would be faster when $N_{\mathrm{D}}$ is larger and when the quality of the initial guess function is better. We then study the structure of the ICI method by expanding its variable space. We also consider how to calculate the excited state by the ICIGSD method. One method is an ICI method aiming at only one exact excited state. The other is to use the higher solutions of the ICIGSD eigenvalues and vectors to compute approximate excited states. The latter method can be improved by extending the variable space outside of GSD. The underlying concept is similar to that of the symmetry-adapted-cluster configuration-interaction (SAC-CI) theory. A similar method of calculating the excited state is also described based on the ICIND method. (C) 2001 American Institute of Physics. [DOI: 10.1063/1.1383032]
\end{abstract}

\section{INTRODUCTION}

The Schrödinger nonrelativistic equation describes much of the world of chemistry. If we can solve this equation with a realistic cost, we can make very precise predictions and its scientific and practical merits are huge. The full CI method gives the exact wave function within a given basis set, but the number of variables involved in this method, $M_{\text {full-CI }}$, easily runs into astronomical figures for basis sets capable of giving accurate results. For singlet molecules with even number of electrons, it is given by

$$
M_{\text {full-CI }}=\frac{1}{m+1}\left(\begin{array}{c}
m+1 \\
\frac{1}{2} N
\end{array}\right)\left(\begin{array}{c}
m+1 \\
\frac{1}{2} N+1
\end{array}\right),
$$

where $m$ is the number of active orbitals, $N$ the number of electrons, and () denotes a binomial coefficient. Table I shows $M_{\text {full-CI }}$ for some typical small molecules assuming a double-zeta basis set. Even for such small molecules, this number is truly astronomical, yet the energy from a doublezeta basis is not accurate enough for chemical predictions.

All the basic physical operators may be written using only one- and two-particle operators. For this reason, the

\footnotetext{
${ }^{a)}$ Author to whom correspondence should be addressed. Electronic mail: hiroshi@sbchem.kyoto-u.ac.jp
}

second-order density matrix $\Gamma^{(2)}\left(1^{\prime} 2^{\prime} \mid 12\right)$ is enough to calculate these properties. ${ }^{1}$ Among other operators, the Hamiltonian, a key operator in quantum mechanics, is written in a second-quantized form as ${ }^{2}$

$$
H=\sum_{p r} v_{p}^{r} a_{r}^{+} a_{p}+\sum_{p q r s} w_{p q}^{r s} a_{r}^{+} a_{s}^{+} a_{q} a_{p},
$$

where the first term is a one-particle operator and the second term is a two-particle operator. In atomic and molecular systems, the electronic Hamiltonian is written in a coordinate representation as

$$
H=\sum_{v}-\frac{1}{2} \Delta_{v}-\sum_{v} \sum_{A} Z_{A} / r_{A v}+\sum_{\mu>v} 1 / r_{\mu v},
$$

where the first kinetic and second nuclear attraction terms are one-electron operators and the third electron repulsion term is a two-electron operator. We therefore expect that the exact wave function $\psi$ that satisfies the Schrödinger equation

$$
(H-E) \psi=0,
$$

i.e., an eigenfunction of such a simple operator, should also have a simple structure: for example, it may be written with a number of variables that is substantially smaller than $M_{\text {full-CI }}$. In this paper, we mainly use the Hamiltonian (1.2) given in a second-quantized form. 
TABLE I. Number of variables for double-zeta basis set.

\begin{tabular}{lcrr}
\hline \hline Molecule & $m$ & \multicolumn{1}{c}{$M_{\text {full-CI }}$} & $M_{\mathrm{GSD}}$ \\
\hline Water & 14 & 1002001 & 8477 \\
Ethylene & 28 & 88385227425 & 143668 \\
Benzene & 72 & $\sim 3.8 \times 10^{34}$ & 6538320 \\
\hline \hline
\end{tabular}

In a previous paper of this series, ${ }^{2}$ which is called Paper I hereafter, one of the authors examined the structure of the exact wave function and showed that it is actually possible to calculate the exact wave function with the number of variables that is equal to the number of general singles and doubles (GSD) substitution operators, $M_{\mathrm{GSD}}$,

$$
M_{\mathrm{GSD}}=m^{2}+\left[\frac{m}{2}(m-1)\right]^{2} \text {. }
$$

We proposed the iterative configuration interaction (ICI) method including $M_{\mathrm{GSD}}$ variables in each iteration step to calculate the exact wave function: it is called the ICIGSD method. The total number of variables in ICIGSD is $n M_{\mathrm{GSD}}$, where $n$ is an iteration number until convergence. Table I shows the number $M_{\mathrm{GSD}}$ for the same molecules. $M_{\mathrm{GSD}}$ is certainly much smaller than $M_{\text {full-CI }}$. In this paper, we continue to study the structure of the exact wave function. We generalize the ICI method from a more general point of view and study the structure of the ICI wave function.

To investigate the structure of the exact wave function, we need the equations that are equivalent to the Schrödinger equation in a necessary and sufficient sense. ${ }^{2}$ Such equations have the same determinative power as the Schrödinger when they are solved appropriately. First, the variational principle

$$
\langle\psi|H-E| \delta \psi\rangle=0,
$$

is equivalent to the Schrödinger equation. The energy of the system $E$ is defined by

$$
\langle\psi|H-E| \psi\rangle=0,
$$

throughout this paper. Second, the equation

$$
\left\langle\psi\left|(H-E)^{2}\right| \psi\right\rangle=0,
$$

is equivalent to the Schrödinger equation, and the following equation:

$$
\langle\psi|(H-E) H| \psi\rangle=0,
$$

together with Eq. (1.7), is also equivalent to the Schrödinger equation. Though we believe that the equivalence of these equations to the Schrödinger equation is well known, proof is given in the Appendix. The density equation, ${ }^{1,3}$ which is equivalent to the Schrödinger equation in the necessary and sufficient sense in the space of the density matrix, was derived based on this theorem. In Paper $\mathrm{I}^{2}$ a theorem is given stating that the following set of equations:

$$
\left\langle\psi\left|(H-E) a_{r}^{+} a_{p}\right| \psi\right\rangle=0,
$$

and

$$
\left\langle\psi\left|(H-E) a_{r}^{+} a_{s}^{+} a_{q} a_{p}\right| \psi\right\rangle=0,
$$

where $E$ is given by Eq. (1.7) and the indices $p, q, r, s$ run through all occupied and unoccupied orbitals, is equivalent to the Schrödinger equation. This theorem has given a basis for constructing a method of calculating the exact wave function with the $M_{\mathrm{GSD}}$ number of variables. ${ }^{2}$ Note that $a_{r}^{+}$and $a_{p}$ in the above equations are the creation and annihilation operators, respectively, defined by using some appropriate orthonormal set of orbitals like Hartree-Fock.

\section{THEOREM}

It is shown in this section that Theorem II-1 of Paper I is a special case of a more general theorem given below.

\section{A. Theorem II-I}

We define a division of the Hamiltonian into $N_{\mathrm{D}}$ parts as

$$
H=\sum_{I=1}^{N_{D}} H_{I} \text {. }
$$

Then, the wave function $\psi$ that satisfies

$$
\left\langle\psi\left|(H-E) H_{I}\right| \psi\right\rangle=0 \quad\left(I=1, \ldots, N_{\mathrm{D}}\right),
$$

with $E$ given by Eq. (1.7) is exact in a necessary and sufficient sense.

\section{Proof}

The necessity is trivial because if $\psi$ satisfies the Schrödinger equation given by Eq. (1.4), it automatically satisfies Eq. (2.2). The sufficiency is also simple. If $\psi$ satisfies Eq. (2.2) for all $I$, we sum them up for all $I$, and using Eq. (2.1) we obtain Eq. (1.9), which implies together with Eq. (1.7) that $\psi$ is exact (QED). by

We define the partial energy $E_{I}$, corresponding to $H_{I}$,

$$
\left\langle\psi\left|H_{I}-E_{I}\right| \psi\right\rangle=0 .
$$

Then, summing up Eq. (2.3) for all $I$, we obtain

$$
\left\langle\psi\left|H-\sum_{I} E_{I}\right| \psi\right\rangle=0,
$$

which implies, comparing to Eq. (1.7)

$$
E=\sum_{I} E_{I}
$$

Using the partial energy $E_{I}$ defined by Eq. (2.3), we obtain the following theorem.

\section{B. Theorem II-2}

The wave function $\psi$ that satisfies Eqs. (2.3), (2.5), and

$$
\left\langle\psi\left|(H-E)\left(H_{I}-E_{I}\right)\right| \psi\right\rangle=0,
$$

for all $I\left(I=1, \ldots, N_{\mathrm{D}}\right)$, is exact in a necessary and sufficient sense.

\section{Proof}

The proof is very similar to that for Theorem II- 1 . The necessity is trivial. The sufficiency is also simple. When we sum up Eq. (2.6) for all $I$, we obtain Eq. (1.8), which implies $\psi$ is exact (QED). 
The two theorems given above have significant and broad utility depending on how we divide the Hamiltonian. We may divide the Hamiltonian into one- and two-electron parts and the one-electron part further into kinetic and different nuclear attraction terms, using the definition of the Hamiltonian given by Eq. (1.3). We may divide $H$ into the Hartree-Fock part and the correlation part. We may divide $H$ into all $p, r$ and $p, q, r, s$ parts, namely into $M_{\mathrm{GSD}}$ parts, using the definition of the Hamiltonian given by Eq. (1.2). In the last case, Eq. (2.2) of Theorem II-1 gives Eqs. (1.10) and (1.11). Then, Theorem II-1 of Paper I is a special case of Theorem II-1 of this section. We note here that how to divide the Hamiltonian is dependent on what expression of the Hamiltonian we use. In this paper, we mainly use the Hamiltonian (1.2) written in a second quantized form.

\section{ICI METHOD—GENERALIZATION}

It is shown in this section that the ICIGSD method given in Paper I is a special case from a group of variational methods that gives the exact wave function with a number of variables from 1 to $M_{\mathrm{GSD}}$ in each iteration step.

We start from a brief explanation of the ICIGSD method. ${ }^{2}$ We define the iterative CI method by the recurrence

$$
\psi_{n}=\left(1+T_{n}\right) \psi_{n-1},
$$

where $T_{n}$ is defined by

$$
T_{n}=\sum_{p r}{ }^{n} C_{p}^{r} a_{r}^{+} a_{p}+\sum_{p q r s}{ }^{n} C_{p q}^{r s} a_{r}^{+} a_{s}^{+} a_{q} a_{p},
$$

using the general singles and doubles substitution operators. The variables in $T_{n},{ }^{n} C_{p}^{r}$, and ${ }^{n} C_{p q}^{r s}$, are determined by the secular equations

$$
\begin{aligned}
& \left\langle\psi_{n}\left|H-E_{n}\right| \psi_{n-1}\right\rangle=0, \\
& \left\langle\psi_{n}\left|\left(H-E_{n}\right) a_{r}^{+} a_{p}\right| \psi_{n-1}\right\rangle=0, \\
& \left\langle\psi_{n}\left|\left(H-E_{n}\right) a_{r}^{+} a_{s}^{+} a_{q} a_{p}\right| \psi_{n-1}\right\rangle=0,
\end{aligned}
$$

which are obtained by applying the variational principle Eq. (1.6) to $\psi_{n}$ given by Eq. (3.1). This procedure is iterated until convergence. When converged, $\psi_{n}$ becomes identical with $\psi_{n-1}$

$$
\psi=\psi_{n}=\psi_{n-1},
$$

and the energy is

$$
E=E_{n}=E_{n-1},
$$

and therefore, Eqs. (3.3), (3.4), and (3.5) become identical to Eqs. (1.7), (1.10), and (1.11), respectively. This means that the converged solution $\psi$ is exact. The number of variables in each iteration step is $M_{\mathrm{GSD}}$, as seen from Eq. (3.2). Since each iteration process is variational, the solution converges from above to the exact solution.

The above ICI method can be generalized based on the theorem given in the preceding section. First, we define the variable operator $S$

$$
S=\sum_{I=1}^{N_{D}} C_{I} H_{I},
$$

corresponding to the division of the Hamiltonian given by Eq. (2.1). $C_{I}\left(I=1, \ldots, N_{\mathrm{D}}\right)$ in Eq. (3.8) are variables to be calculated. We now assume a recurrence

$$
\psi_{n}=\left(1+S_{n}\right) \psi_{n-1},
$$

and determine the variables ${ }^{n} C_{I}$ variationally at each step. The label $n$ on ${ }^{n} C_{I}$ and $S_{n}$ denotes the iteration number. Applying the variational principle to $\psi_{n}$, we get the secular equation

$$
\begin{aligned}
& \left\langle\psi_{n}\left|H-E_{n}\right| \psi_{n-1}\right\rangle=0, \\
& \left\langle\psi_{n}\left|\left(H-E_{n}\right) H_{I}\right| \psi_{n-1}\right\rangle=0 \quad\left(I=1, \ldots, N_{D}\right) .
\end{aligned}
$$

Note that the energy of the $n$th iteration $E_{n}$ satisfies

$$
\left\langle\psi_{n}\left|H-E_{n}\right| \psi_{n}\right\rangle=0,
$$

as well as Eq. (3.10). It is easily derived from Eqs. (3.8)(3.11). When converged, $\psi_{n}=\psi_{n-1}=\psi$ and $E_{n}=E_{n-1}=E$, and we have Eq. (1.7) and

$$
\left\langle\psi\left|(H-E) H_{I}\right| \psi\right\rangle=0 .
$$

Summing up Eq. (3.13) for all $I$, we obtain

$$
\langle\psi|(H-E) H| \psi\rangle=0,
$$

which combined with Eq. (1.7) shows that the converged solution is exact. We note that this procedure is valid not only to the ground state, but also to the excited state, as discussed in more detail later in this paper.

We can formulate the above iterative method in a slightly different way. We define the variable operator $S$ as

$$
S=\sum_{I=1}^{N_{D}} c_{I}\left(H_{I}-E_{I}\right)
$$

using $E_{I}$ defined by Eq. (2.3). The ICI recurrence formula for this variable operator $S$ is also given by Eq. (3.9), and applying the variational principle, we get after convergence

$$
\left\langle\psi\left|(H-E)\left(H_{I}-E_{I}\right)\right| \psi\right\rangle=0,
$$

instead of Eq. (3.14). Summing up Eq. (3.16) for all $I$, we obtain

$$
\left\langle\psi\left|(H-E)^{2}\right| \psi\right\rangle=0,
$$

which again shows that the converged solution is exact. Since the ICI method defined by the recurrence given by Eq. (3.9) with the $S$ operator given by Eq. (3.8) or (3.15) includes $N_{\text {D }}$ variables in each iteration step, we call this the ICIND method and it is summarized as follows.

ICIND method: when the Hamiltonian is divided into $N_{\text {D }}$ parts as Eq. (2.1), we define the variable operator $S$ by Eq. (3.8) or (3.15). Then, we can formulate the ICI method by Eq. (3.9), where the number of variables in each iteration step is $N_{\mathrm{D}}$, and when converged, this method gives the exact wave function. In the converging process, the ICI solution approaches from above the exact solution, since each iteration step is variational.

We discuss two extreme cases of the ICI method. First, when we do not divide the Hamiltonian, the ICI recurrence formula Eq. (3.9) is written as 


$$
\psi_{n}=\left(1+{ }^{n} \mathrm{CH}\right) \psi_{n-1},
$$

or

$$
\psi_{n}=\left[1+{ }^{n} c\left(H-E_{n}\right)\right] \psi_{n-1},
$$

depending on whether we take Eq. (3.8) or (3.15) as a $S$ operator. The number of variables of this method in each iteration step is only one, so this is referred to as the ICION (one) method or simplest ICI (SICI) method. A similar method has been frequently advocated and the convergence property has been discussed. ${ }^{4}$

Second, when we divide the Hamiltonian into each $p, r$ one-electron part and each $p, q, r, s$ two-electron part, $N_{\mathrm{D}}$ is equal to $M_{\mathrm{GSD}}$ and the operator $S$ is equivalent to the operator $T$ as

$$
\begin{aligned}
S & =\sum_{p q} c_{p}^{r} v_{p}^{r} a_{r}^{+} a_{p}+\sum_{p q r s} c_{p q}^{r s} w_{p q}^{r s} a_{r}^{+} a_{s}^{+} a_{q} a_{p} \\
& =\sum_{p q} C_{p}^{r} a_{r}^{+} a_{p}+\sum_{p q r s} C_{p q}^{r s} a_{r}^{+} a_{s}^{+} a_{q} a_{p}=T .
\end{aligned}
$$

Therefore, this is the ICIGSD method proposed in Paper I of this series. ${ }^{2}$

Another interesting division of the Hamiltonian is the singles division that gives the ICIGS (general singles) method. For example, we may divide $H$ into $M_{s}$ number of "singles" operators $H_{p}^{r}$ as

$$
H=\sum_{p r} H_{p}^{r},
$$

where

$$
\begin{aligned}
& H_{p}^{r}=a_{r}^{+} h_{p}^{r} a_{p}, \\
& h_{p}^{r}=v_{p}^{r}+\sum_{q s} a_{s}^{+} w_{p q}^{r s} a_{q} .
\end{aligned}
$$

Then, correspondingly, we have the operator $S$ as

$$
S=\sum_{p r} C_{p}^{r} H_{p}^{r} .
$$

The secular equation for this method is written from Eqs. (3.10) and (3.11) as

$$
\begin{aligned}
& \left\langle\psi_{n}\left|H-E_{n}\right| \psi_{n-1}\right\rangle=0, \\
& \left\langle\psi_{n}\left|\left(H-E_{n}\right) H_{p}^{r}\right| \psi_{n-1}\right\rangle=0, \quad E_{n} \geqslant E,
\end{aligned}
$$

where $E$ is the exact energy. Since the wave function of this method given by Eq. (3.9) with $S$ defined by Eq. (3.24) includes only $M_{s}$ variables, we may regard Eq. (3.25) as being related to the basic existence theorem ${ }^{5}$ of the density functional theory (DFT), ${ }^{6}$ like Hohenberg-Kohn theorem ${ }^{4}$ and others. ${ }^{7-9}$ Since there are many different ways of dividing the Hamiltonian into $M_{s}$ operators, it is interesting to speculate whether such a singles method could be rewritten using electron density or the first-order density matrix alone.

In actual calculations, the convergence property of the ICIND method is very important. We believe that in principle, this method should converge. As easily seen from Eqs. (3.18) and (3.19), the SICI method is related to the power method and the Lanczos method in the eigenvalue problem. ${ }^{10}$ It converges to largest absolute eigenvalue, but by modification, it is made to converge to the ground state. Since

$$
\delta \frac{\left\langle\psi_{n}|H| \psi_{n}\right\rangle}{\left\langle\psi_{n} \mid \psi_{n}\right\rangle}=\left\langle\psi_{n}\left|H-E_{n}\right| \psi_{n}\right\rangle,
$$

when $\left\langle\psi_{n} \mid \psi_{n}\right\rangle=1$, Eq. (3.19) is equivalent to a variational step along the gradient to the average energy. The convergence rate would be accelerated when we use larger number of variational parameters, and this is realized by using the ICIND and the ICIGSD method, where the number of variables is $N_{\mathrm{D}}$ and $M_{\mathrm{GSD}}$, respectively. Thus, since SICI converges, in principle, the ICIND and ICIGSD methods should also converge, in principle.

In actual calculations, the convergence property is dependent on other technical matters. Since the convergence rate would be faster when $N_{\mathrm{D}}$ is larger, it is desirable to use the largest possible $N_{\mathrm{D}}$ for realizing fast convergence. Note further that the number of the divisions, $N_{\mathrm{D}}$, is not necessarily kept constant during the iteration process. By changing $N_{\mathrm{D}}$, we can adjust the labor necessary for diagonalizing the matrices. Allowing such a flexibility in the computational algorithm would be useful in applications of the ICIND method and some discussions are given in Sec. IV.

Another factor affecting the convergence rate is the quality of the initial function $\psi_{0}$. An appropriate choice of $\psi_{0}$ in symmetry and in the nature is an important prerequisite: the better the quality of the initial function $\psi_{0}$, the faster the convergence. Note that if we start from a single determinant initial function, the coefficients for excitations higher than $2 n$-fold will be kept zero until iteration $n$. The energy may converge rapidly, but the convergence rate may be dependent on the size of the molecule. It would be useful to prepare the initial function by the method of a high-performance algorithm along the line of the ICIND method, or by the method now available. For example, we may adopt a nonvariational procedure for preparing the initial guess, or we may adopt, for example, the conventional CCSD (coupled cluster singles and doubles) for $\psi_{0}$. In the latter case, all levels of excitations are included in $\psi_{0}$ from the beginning in an approximate way, so that the convergence rate would be faster than using the Hartree-Fock initial function.

\section{EXPANSION OF THE VARIABLE SPACE OF THE ICI METHOD}

We consider in this section an expansion of the variable space of the ICI method and see what happens by such an expansion. We consider two different expansions: one is the expansion from the ICIGSD method $\left(N_{\mathrm{D}}=M_{\mathrm{GSD}}\right)$ to include triple and higher operators, and the other is from the intermediate case (ICIND) where $N_{\mathrm{D}}$ is between 1 and $M_{\mathrm{GSD}}$.

First, we consider the expansion from the ICIGSD method. We define the general operator $G$ by

$$
G=H+L
$$

where $H$ is the Hamiltonian of the system and $L$ is some operator defined later. Here, note that we are interested in the eigenfunction of $H$, not of $G$. So, $L$ is not a perturbation. We rewrite the Hamiltonian operator given by Eq. (1.2) as 


$$
H=\sum_{p r} v_{p}^{r} a_{r}^{+} a_{p}+\sum_{p q r s} w_{p q}^{r s} a_{r}^{+} a_{s}^{+} a_{q} a_{p}=\sum_{i}^{M_{\mathrm{GSD}}} x_{i} t_{i},
$$

where $x_{i}$ represents the integrals, $v_{p}^{r}$ and $w_{p q}^{r s}$, and $t_{i}$ represents the substitution operators, $a_{r}^{+} a_{p}$ and $a_{r}^{+} a_{s}^{+} a_{q} a_{p}$. Similarly, the operator $L$ is also expressed as

$$
L=\sum_{j} y_{j} r_{j},
$$

where $y_{j}$ is some number and $r_{j}$ the substitution operator. Corresponding to $H$ of Eq. (4.2), we define the GSD operator $T$ as

$$
T=\sum_{p r} C_{p}^{r} a_{r}^{+} a_{p}+\sum_{p q r s} C_{p q}^{r s} a_{r}^{+} a_{s}^{+} a_{q} a_{p}=\sum_{i}^{M_{\mathrm{GSD}}} c_{i} t_{i},
$$

and similarly, corresponding to $L$ we define the variable operator $R$ as

$$
R=\sum_{j} d_{j} r_{j},
$$

where $c_{i}$ and $d_{j}$ represent unknown variables. Thus, corresponding to $G$ we have defined the variable operator

$$
U=T+R \text {. }
$$

We now consider the recurrence

$$
\psi_{n}=\left(1+U_{n}\right) \psi_{n-1} \text {. }
$$

Applying the variational principle given by Eq. (1.6) to $\psi_{n}$, we get

$$
\begin{aligned}
& \left\langle\psi_{n}\left|H-E_{n}\right| \psi_{n-1}\right\rangle=0, \\
& \left\langle\psi_{n}\left|\left(H-E_{n}\right) t_{i}\right| \psi_{n-1}\right\rangle=0, \\
& \left\langle\psi_{n}\left|\left(H-E_{n}\right) r_{j}\right| \psi_{n-1}\right\rangle=0,
\end{aligned}
$$

and when converged, $\psi_{n}=\psi_{n-1}=\psi$ and $E_{n}=E_{n-1}=E$, we obtain

$$
\begin{aligned}
& \langle\psi|H-E| \psi\rangle=0, \\
& \left\langle\psi\left|(H-E) t_{i}\right| \psi\right\rangle=0 . \\
& \left\langle\psi\left|(H-E) r_{j}\right| \psi\right\rangle=0,
\end{aligned}
$$

for all $i\left(1 \leqslant i \leqslant M_{\mathrm{GSD}}\right)$ and $j$. Equations (4.11) and (4.12) guarantee that the solution $\psi$ is exact, but Eq. (4.13) has nothing to do with the proof that guarantees the exactness of $\psi$. Namely, the operators $L$ and $R$ do not affect the final solution.

We classify the operator $R$ into two cases: one is the case where the operator $R$ is expressed within singles and doubles and the other is the case where the operator $R$ belongs to the outside of the singles and doubles, namely to triples and higher operators.

When we add some physical operator $L$ to the Hamiltonian as in Eq. (4.1), the corresponding $R$ operator belongs to the general singles and doubles space, since any physical operators can be expanded by the GSD operators $\left\{a_{r}^{+} a_{p}, a_{r}^{+} a_{s}^{+} a_{q} a_{p}\right\}$. This means that the addition of the variable operator $R$ as Eq. (4.6) is redundant to the already existing GSD variable space represented by the $T$ operator given by Eq. (4.4). Then, in this case the addition of the $L$ and $R$ operators has no effect at all on either the iteration process or the final result.

On the other hand, when $R$ includes the operators from triple to $N$-ple excitation or substitution operators, it enlarges the variable space to be larger than $M_{\mathrm{GSD}}$ and certainly affects the variational process, though it does not affect the converged solution. Actually, when $R$ includes all triple to $\mathrm{N}$-ple excitation operators, the above method gives full CI and the iteration converges at once. ${ }^{2}$

We next consider the expansion of the variable space from the intermediate ICIND method in which the number of variables is $N_{\mathrm{D}}$ which is between 1 and $M_{\mathrm{GSD}}$. In this case the definition of the operator $G$ is the same, but that of $U$ is different from Eq. (4.6) and is given by

$$
U=S+R,
$$

using the $S$ operator given by Eq. (3.8). Correspondingly, Eqs. (4.9) and (4.12) are rewritten as

$$
\left\langle\psi_{n}\left|\left(H-E_{n}\right) H_{I}\right| \psi_{n-1}\right\rangle=0,
$$

and

$$
\left\langle\psi\left|(H-E) H_{I}\right| \psi\right\rangle=0,
$$

respectively. Since the operator space $\left\{H_{I}\right\}$ is incomplete within the GSD space, the operator $L$ that is physical or that belongs to the GSD space cannot generally be expanded by $\left\{H_{I}\right\}$. Therefore, the addition of the $R$ operator may expand the variable space and may affect the iteration process, though of course, the final converged solution is unaffected. Similarly, when the $R$ operator includes the operators from triple to $\mathrm{N}$-ple excitation and substitution operators, it would also affect the variational process, though again, it does not affect the final solution. When the $R$ operator affects the variational process.

In summary, as only the Hamiltonian $H$ defines the system, the additional operator $L$ has nothing to do with the solution of the ICI method. Only its variable counterpart $R$ plays a role for controlling the convergence of the iteration process. Furthermore, by expanding the variable operator space by $R$, we can calculate higher excited states by the method described below.

\section{EXCITED STATES BY THE ICIGSD AND ICIND METHODS}

As briefly described in Paper I, the ICIGSD method is applicable not only to the ground state but also to the excited state. We describe in this section how to calculate the excited states by the ICIND method. Since the ICIGSD method is an important extreme, we first discuss the excited-state calculations based on the ICIGSD method. We give three methods called method A, B, and C.

First, since the ICIGSD method is a one-state theory, as would be clear from the Appendix, we calculate the excited state iteratively as follows. We refer to this method as method A. Suppose that we are interested in the second state (first excited state). Then, we take the second state in the initial guess, and then continue to choose only the "second" state in the iteration process. This would lead to convergence 
to the second state, which should be exact from the theorem. Note, however, that second in this procedure is only the number in the initial guess and does not mean that the state is the true second state. The "third" state in the initial guess may become lower than the second state as often occurs in an ordinary iterative diagonalization process like Davidson's. ${ }^{11}$

In method B, we utilize the higher-energy solutions simultaneously obtained in the ICIGSD calculation of the ground state as the excited states. At the convergence of the ICI calculation, we obtain the exact ground state $\psi^{g}$ and at the same time, the higher-energy solutions $\psi^{K}$ of the same secular equation that are written as

$$
\psi^{K}=\sum_{i=1}^{M_{\mathrm{GSD}}} c_{i}^{K} t_{i}\left|\psi^{g}\right\rangle,
$$

where $t_{i}$ represents the singles and doubles substitution operators as defined by Eq. (4.4). Since $\psi^{K}$ are the solutions of the same secular equation as for $\psi^{g}$, they satisfy

$$
\left\langle\psi^{K} \mid \psi^{g}\right\rangle=0, \quad\left\langle\psi^{K}|H| \psi^{g}\right\rangle=0
$$

and

$$
\left\langle\psi^{K} \mid \psi^{K^{\prime}}\right\rangle=\delta_{K K^{\prime}}, \quad\left\langle\psi^{K}|H| \psi^{K^{\prime}}\right\rangle=E_{K} \delta_{K K^{\prime}} .
$$

Since Eqs. (5.2) and (5.3) are important necessary conditions of the excited states, we may take $\psi^{K}$ as representing the excited states of $\psi^{g}$.

Now, what is the quality of the excited states calculated by method B? The excited functions $\psi^{K}$ of method B are generated by applying the GSD operators to the exact ground state $\psi^{g}$ as expressed by Eq. (5.1). It is also interesting to consider a set of functions $\varphi_{i}$ defined by

$$
\varphi_{i}=\sum_{j} d_{j i} u_{j}\left|\psi^{g}\right\rangle
$$

where $\left\{u_{j}\right\}$ represents the operators from triple to $N$-ple excitation operators. Since $\psi^{g}$ is exact, it satisfies

$$
\left\langle\varphi_{i}|H-E| \psi^{g}\right\rangle=0 .
$$

However, $\left\{\psi^{K}\right\}$ obtained simultaneously with $\psi^{g}$ do not necessarily satisfy the similar relation to Eq. (5.5), namely

$$
\left\langle\varphi_{i}|H-E| \psi^{K}\right\rangle \neq 0,
$$

because $\varphi_{i}$, which is linearly independent from $\psi^{K}$, are not included in the secular equation which gives $\psi^{g}$. This means that the excited-state functions $\left\{\psi^{K}\right\}$ of method B obtained simultaneously with $\psi^{g}$ are not exact, though they satisfy Eqs. (5.2) and (5.3).

The quality of the excited states by method B is therefore improved by extending the variable space as

$$
\psi^{L}=\left(\sum_{i} c_{i}^{L} t_{i}+\sum_{j} d_{j}^{L} u_{j}\right)\left|\psi^{g}\right\rangle
$$

where $\left\{t_{i}\right\}$ represents GSD operators and $\left\{u_{j}\right\}$ the operators from triple to $N$-ple excitation operators. By further diagonalizing (only once) the secular equation corresponding to Eq. (5.7), we can improve the quality of the excited state, up to the exact limit. Furthermore, by this method, we can cal- culate higher multiple excitations which were rather poor or were not calculated in method B. We refer to this method as method $\mathrm{C}$.

The methods of calculating the excited state described in this section have some similarity to already existing methods. ${ }^{12}$ The iterative method $\mathrm{A}$ is similar to the symmetry adapted cluster (SAC) method ${ }^{13,14}$ and the MR(multireference)-SAC method ${ }^{15,16}$ for excited and openshell electronic states. Method B is similar to the SAC-CI SD- $R$ method, ${ }^{12,17}$ and method $\mathrm{C}$ to the SAC-CI general- $R$ method. ${ }^{18}$ In the SAC-CI SD- $R$ method, the SAC-CI excitation operators $R$, which are similar to the operator $t_{i}$ in Eq. (5.1), are limited within singles and doubles, while in the general- $R$ method, they are taken not only from the SD space but also from triple to higher excitation space. The SAC-CI $\mathrm{SD}-R$ method is accurate for describing ordinary singleelectron excitation processes, but insufficient for describing the multiple-electron excitation processes, ${ }^{18}$ for which the SAC-CI general- $R$ method is accurate. ${ }^{19}$

It is very interesting which of the three methods given in this section is most efficiently accurate for the study of excited states of atoms and molecules. Probably method B, which is easiest among the three, would be most useful for ordinary excitation processes. Needless to say, the expression of the excited state by Eqs. (5.1) and (5.7) represents the transferability of electron correlations between ground- and excited states. Further, by including the operators belonging to different symmetries and the ionization and electron attachment operators in the operators $t_{i}$ and $u_{j}$, we can also describe different excited states (e.g., triplet excited states) and ionized and electron attached states as well as the excited states having the same symmetry as the ground state, just as in the SAC-CI method. ${ }^{12,17}$

It is also possible to calculate the excited states in the ICIND formalism. Method A is essentially the same as in the ICIGSD case. We use an initial guess function $\psi_{0}$ having the symmetry and the electronic structure desired for the excited state to be calculated, and choose to keep only such functions until convergence. Again, the converged solution should be exact. In the case of method B, the quality of the excited states obtained simultaneously with the ground state would be worse than that for the ICIGSD method, when the variational space for the excited states of the ICIND method is smaller than that of the ICIGSD method. The quality of the excited states can be improved and the range of the excitations calculated are expanded by extending the variable space from ND to GSD or even to include triple to N-ple excitation operators. This method corresponds to method $\mathrm{C}$ of the above paragraphs. We believe from the experiences of the SAC/SAC-CI calculations that the ICIGSD method, followed by method B in particular, is a useful method for calculating the ground- and excited states of molecules and molecular systems.

\section{CONCLUSION}

The Schrödinger equation itself should be correct even if the basic operator involves up to $N$-particle interaction terms, but in reality, the Hamiltonian operator involves only up to two-particle interaction terms. Since the exact wave function 
is an eigenfunction of the Hamiltonian that has such a simple structure, the exact wave function itself should also have a simple structure. This is a basic philosophy underlying the present series of studies. We study the structure of the exact wave function under the expectation that it should be simple and hopefully beautiful.

In Paper $\mathrm{I}^{2}$ we presented a theorem that implies an existence of the general singles and doubles description of the exact wave function. Namely, the number of variables necessary to describe the exact wave function would be reduced to $M_{\mathrm{GSD}}$ given by Eq. (1.5) instead of $M_{\text {full-CI }}$ given by Eq. (1.1). We examined both exponential and linear expansion ansätz and proposed the ICIGSD method as a method of calculating the exact wave function with the GSD number of variables.

In this paper, we have generalized Theorem II- 1 of Paper I: when we divide the Hamiltonian into $N_{D}$ parts, we have a set of $N_{\mathrm{D}}$ equations that is equivalent to the Schrödinger equation in the necessary and sufficient sense. Based on this theorem, the ICI method was generalized to calculate the exact wave function with $N_{\mathrm{D}}$ number of variables where $N_{\mathrm{D}}$ ranges from 1 to $M_{\mathrm{GSD}}$ where $M_{\mathrm{GSD}}$ is the case of the ICIGSD method. The simplest case with $N_{\mathrm{D}}=1$ (ICION or SICI) is very interesting, and the general singles case where $N_{\text {D }}$ is the number of general single substitution operators (ICIGS) is also interesting. In actual ICI calculations, the convergence rate would be faster when $N_{\mathrm{D}}$ is larger and when the quality of the initial function is better. Further, $N_{\mathrm{D}}$ is not necessarily kept constant throughout the ICI calculations.

We studied the structure of the ICI method by first expanding its variable space. GSD is a good special case because the GSD operator space is complete in the sense that it can expand any physical operators. If we extend the variable space outside of GSD by including some triple to $N$-ple excitation operators, the variational process would be accelerated though the final solution should be the same.

We considered how to calculate the excited state by the ICIGSD method. We proposed three methods. One is an iterative method aiming at only one excited state, and the other is the method utilizing the higher-energy solutions obtained simultaneously with the ground state. The first method gives the exact solution, but the second one is not exact, though the solutions satisfy the orthogonality and Hamiltonian orthogonality with the calculated exact ground state. The latter method has some similarity to the SAC-CI SD- $R$ method. By extending the variable space after obtaining the exact ground state, we can improve the excited state: this method is similar to the SAC-CI general- $R$ method for calculating the excited states, in particular, the multiple-electron excited states. Similar methods are also described based on the ICIND method.

\section{ACKNOWLEDGMENT}

This study has been supported financially by the special fund from the Ministry of Education, Science, Culture, and Sports of Japan.

\section{APPENDIX}

We here prove that each of Eqs. (1.8) and (1.9) with $E$ defined by Eq. (1.7) is equivalent to the Schrödinger equation in a necessary and sufficient sense.

The necessity is trivial: if $\psi$ satisfies Eq. (1.4), it satisfies Eqs. (1.8) and (1.9). We prove the sufficiency. We assume that we have the solutions of the Schrödinger equation as

$$
H \Psi_{n}=E_{n} \Psi_{n} .
$$

Since $\left\{\Psi_{n}\right\}$ forms a complete set of eigenfunctions of the Hamiltonian, we can expand our $\psi$ as

$$
\psi=\sum_{n} C_{n} \Psi_{n} .
$$

Inserting Eq. (A2) into our expression given by Eq. (1.8), we have

$$
\begin{aligned}
0=\left\langle\psi\left|(H-E)^{2}\right| \psi\right\rangle & =\sum_{n, m} C_{n}^{*} C_{m}\left\langle\Psi_{n}\left|(H-E)^{2}\right| \Psi_{m}\right\rangle \\
& =\sum_{n}\left|C_{n}\right|^{2}\left(E_{n}-E\right)^{2} .
\end{aligned}
$$

Since all terms in the last sum are non-negative, Eq. (A3) stands only when all $C_{n}$ are zero except for one case $n=i$, for which $E=E_{i}$ and $C_{i} \neq 0$. From the normalization condition, $C_{i}=1$, and therefore, from Eq. (A2), $\psi=\Psi_{i}$. Namely, $\psi$ is an eigenfunction of the Schrödinger equation. It can be either ground state or excited state. When $E_{i}$ is degenerate, $\psi$ becomes a linear combination of one set of the degenerate eigenfunctions of $E_{i}$ of the Schrödinger equation. Thus, the sufficiency of Eq. (1.8) is proved. Equation (1.9) is easily obtained from Eq. (1.8) by using Eq. (1.7) (QED).

${ }^{1}$ H. Nakatsuji, in Many-Electron Densities and Reduced Density Matrices edited by J. Cioslowski (Kluwer Academic, New York, 2000).

${ }^{2}$ H. Nakatsuji, J. Chem. Phys. 113, 2949 (2000).

${ }^{3}$ H. Nakatsuji, Phys. Rev. A 14, 41 (1976).

${ }^{4}$ See, for example, E. R. Davidson, Comput. Phys. Commun. 53, 1 (1989); W. A. Berger, H. G. Miller, and R. M. Dreizler, J. Phys. A 10, 1089 (1977); J. H. van Lenthe and P. Pulay, J. Comput. Chem. 11, 1164 (1990).

${ }^{5}$ P. Hohenberg and W. Kohn, Phys. Rev. 136, B864 (1964); H. Huang, Q. Xie, Z. Cao, Z. Li, Z. Yue, and L. Ming, J. Chem. Phys. 110, 3703 (1999); H. Huang, X. Zeng, and L. Ming, ibid. 112, 5257 (2000); M. G. Marmorino, ibid. 114, 2513 (2001).

${ }^{6}$ R. G. Parr and W. Yang, Density-Functional Theory of Atoms and Molecules (Springer, New York, 1989).

${ }^{7}$ H. Nakatsuji and R. G. Parr, J. Chem. Phys. 63, 1112 (1975).

${ }^{8}$ M. Levy, Proc. Natl. Acad. Sci. U.S.A. 76, 6062 (1979).

${ }^{9}$ A. Klein and R. M. Dreizler, Phys. Rev. A 57, 2485 (1998).

${ }^{10}$ J. H. Wilkinson, The Algebraic Eigenvalue Problem (Oxford University Press, Oxford, 1965).

${ }^{11}$ E. R. Davidson, J. Comput. Phys. 17, 87 (1975).

${ }^{12} \mathrm{H}$. Nakatsuji, in Computational Chemistry, Reviews of Current Trends, edited by J. Leszczynski (World Scientific, Singapore, 1997), Vol. 2, pp. $62-124$.

${ }^{13}$ H. Nakatsuji and K. Hirao, J. Chem. Phys. 68, 2053 (1978).

${ }^{14}$ K. Hirao and H. Nakatsuji, Chem. Phys. Lett. 79, 292 (1981).

${ }^{15}$ H. Nakatsuji, J. Chem. Phys. 83, 713 (1985).

${ }^{16}$ H. Nakatsuji, Theor. Chim. Acta 71, 201 (1987).

${ }^{17}$ H. Nakatsuji, Chem. Phys. Lett. 59, 362 (1978); 67, 329 (1979); 67, 334 (1979).

${ }^{18}$ H. Nakatsuji, Chem. Phys. Lett. 177, 331 (1991).

${ }^{19}$ M. Ehara, P. Tomasello, J. Hasegawa, and H. Nakatsuji, Theor. Chem. Acc. 102, 161 (1999). 